\title{
A Case of Papillary Edema Revealing a Cerebral Abscess with Toxoplasma gondii, at the Maurice Ophthalmological Clinic
}

\section{Maxime Dantouma Sovogui ${ }^{1,2 *}$, Mohamed Cherif ${ }^{1,3}$, Christophe Zoumanigui $^{2}$ and Fodé Amara Traore ${ }^{1,4}$}

${ }^{1}$ Faculty of Health Sciences and Techniques of the University of Conakry, Republic of Guinea

${ }^{2}$ Maurice Ophthalmological Clinic, Republic of Guinea

3Neurosurgery Department of the Sino-Guinean Friendship Hospital, Republic of

Guinea

${ }^{4}$ Department of Infectious and Tropical Diseases of donka National Hospital,

Republic of Guinea

*Corresponding Author: Maxime Dantouma Sovogui, Faculty of Health Sciences and Techniques of the University of Conakry, Republic of Guinea.
Received: December 14, 2021

Published: January 20, 2022

(C) All rights are reserved by Maxime

Dantouma Sovogui., et al.

\begin{abstract}
Objective: To describe the ophthalmologic manifestations in the setting of a Toxoplasma gondii brain abscess revealed by papillary edema at the Maurice ophthalmologic clinic in Guinea. Because of its gravity

Patient and Observation: We report here the case of a 17-year-old female student diagnosed with Toxoplasma gondii brain abscess revealed by papillary edema. The symptomatology was dominated by: visual blurring, headache, vomiting, lethargy and left hemiparesis. Stage II papillary edema has been found in fundoscopy. The patient was managed in collaboration with the neurosurgery and tropical infectious diseases departments. She benefited from: Acetazolamide $250 \mathrm{mg}$, Dexamethasone $4 \mathrm{mg}$, Cotrimoxazole and Paracetamol. A relay was conducted namely: Cortancyl $20 \mathrm{mg}$, cotrimoxazole $960 \mathrm{mg}$ and Acetazolamide. An optical correction has been introduced. The evolution was marked by an improvement in the clinical condition with decreased abscess on computed tomography. Continued treatment was observed for two (2) months in a row.

Conclusion: When faced with papillary edema associated with headache and projectile vomiting in an infectious context, a Toxoplasma gondii brain abscess should be suspected until proven otherwise. The positive diagnosis is based on the bundles of clinical and imaging arguments for which the ophthalmologist plays an important role. In this patient, papillary edema was suggestive of the underlying pathology and prompted us to do more investigation.
\end{abstract}

Keywords: Papillary Edema; Brain Abscess; Guinea

\section{Introduction}

Papillary edema (PAP) is the manifestation of axonal suffering of the ganglion cells of the optic nerve in their pre-laminar portion [1]. It is a fluid and/or axonal swelling of the head of the optic nerve due to a blockage of the axoplasmic flow at the level of the screened slide [2]. The positive diagnosis is made at the fundus and is based on the presence: of a blurring of the papillary edges with poor vascular visibility, an edematous aspect of the nerve fibers of the papil- 
la that is more or less protruding. A combination of hemorrhages and peripapillary exudates is possible [3]. Cerebral toxoplasmosis is a brain infection due to Toxoplasma gondii which is a ubiquitous intracellular protozoan [4]. In its abcede form, it is characterized by a collection of pus in the brain, associated with peri-lesional edema that will increase intracranial pressure (ICP) [5]. PAP is formed as a result of this intracranial hypertension [6]; it would be the consequence of the circulation disorder of the cerebrospinal fluid (CSF) in the subarachnoid space surrounding the optic nerves, related to a sectorization of this space [7]. We report the case of a 17-yearold patient who presented with papillary edema in front of signs of neurological involvement. The aim of this work is to describe the ophthalmological manifestations in the context of a brain abscess revealed by papillary edema at the Maurice Ophthalmological Clinic in Guinea.

\section{Patient and Observation}

This is Miss F. aged 17, student, received on June 10, 2021 for: Blurred vision from afar, pulsating headaches rebellious to the usual analgesics, projectile vomiting, lethargy, weakness of the left hemibody, of poorly elucidated evolution. She would have passed in several clinics of the place where the diagnosis of rheumatic fever was always made and then treated with antibiotics of the class of beta-lactams (Ampicillin 1 gram injectable) and cephalosporin of $3^{\text {rd }}$ generation (Ceftriaxone 1 gram injectable) for several weeks, without favorable follow-up. Given the appearance of unusual visual blurring, his parents decided to do an ophthalmological consultation. She was not immunocompromised.

\section{At the ophthalmological examination}

The visual acuity without correction was $8 / 10^{\text {th }}$ in both eyes, then at the pinhole equal to $10 / 10^{\text {th }}$ in both eyes. Healthy appendages, positive photomotor reflex, deep anterior chambers, fundus (FO) highlighted bilateral stage II papillary edema. Neurological examination revealed left hemiparesis with segmental muscle strength rated at $3 / 5$.

At this point, we are thinking of an intracranial hypertension syndrome.

As diagnostic hypotheses, we think of:

- Intracranial vascular causes (hematoma, cerebral infarction, cerebral thrombophlebitis, stroke);

- Intracranial expansive process (tumor, meningiomas);
- Idiopathic cause;

- $\quad$ Bacterial, viral or fungal brain abscess.

Retinography, papillary OCT and automated visual field (CV) performed showed respectively stage II bilateral papillary edema and significant narrowing of the visual field beyond 75\% (Figures 2, 3 and 4).

Diffusion imaging, T1 GADO has highlighted a cystic fronto-parietal intra axial expansive process with engagement under falcique, evoking cystic glioblastoma in the first place; a parasitic origin such as toxoplasma that cannot be formally eliminated if the patient is immunocompromised (Figure 1).
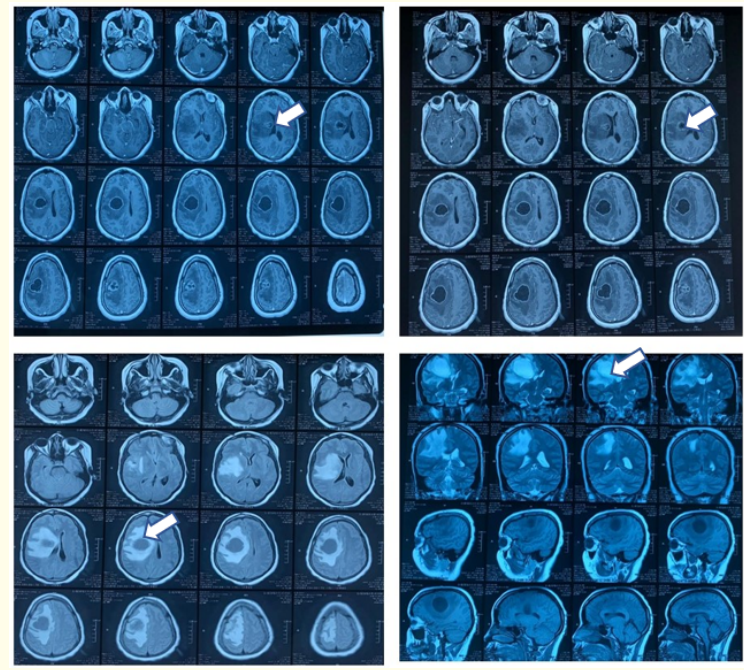

Figure 1: Brain MRI before and after injection of contrast medium highlighting the brain abscess. The arrow indicates the site of the brain abscess.

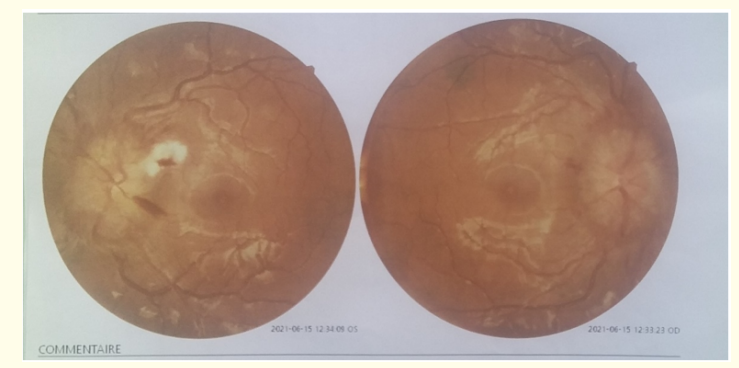

Figure 2: Retinography showing stage II bilateral papillary edema. 

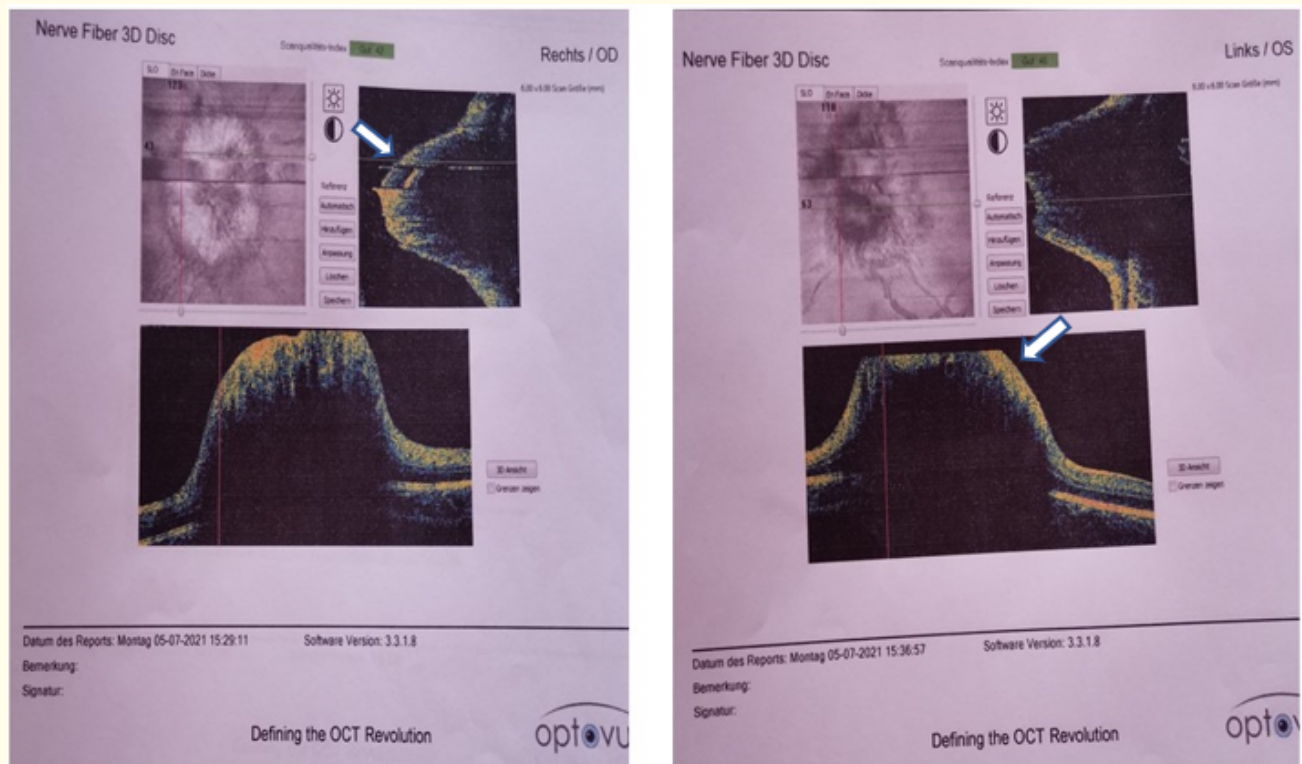

Figure 3: Papillary OCT showing stage II bilateral papillary edema. The arrow indicates the elevation of the papilla.
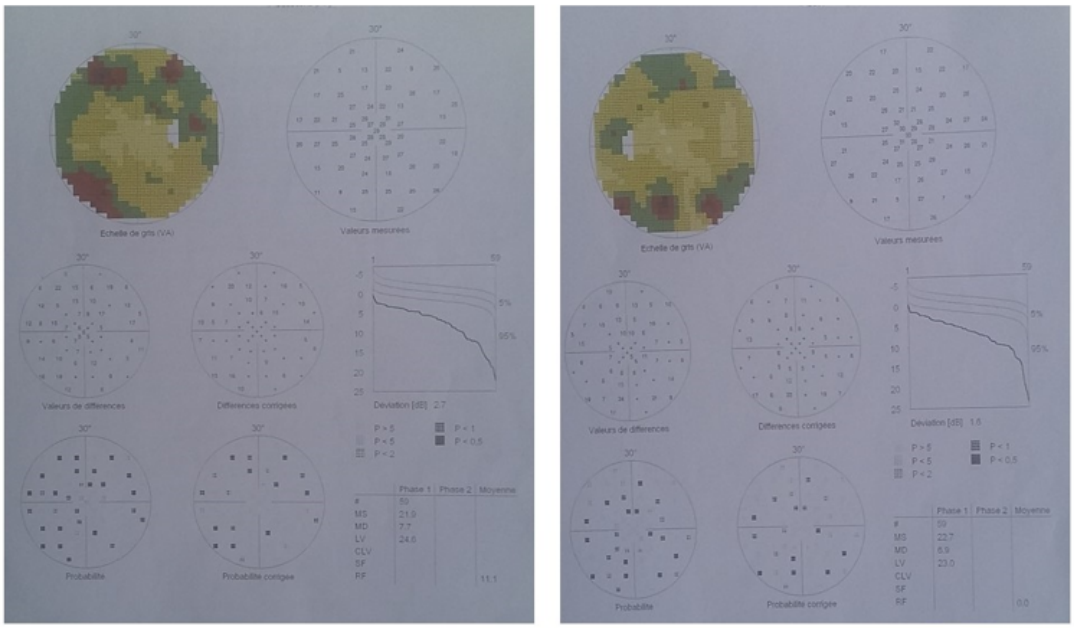

Figure 4: The automated CV highlighting a significant narrowing of the visual field beyond 75\%.

The patient was referred to the neurosurgery department of the Sino-Guinean Friendship Hospital where blood biological examinations (blood count, serology and biochemistry) carried out which highlighted hyperleukocytosis at $11,000 / \mathrm{mm}^{3}$, a positive CRP, the toxoplasmic serology performed was positive by EIA with the fol- lowing antibody levels: IgG: $0.90 \mathrm{IU} / \mathrm{ml}$ and IgM: 0.00. Confirming an ongoing infectious process (Toxoplasmosis). She was not immunocompromised.

Faced with these bundles of arguments, we retain the diagnosis of brain abscess associated with ametropia. 
The patient was then referred to the infectious and tropical diseases department of the Donka National Hospital where she was subjected to the following treatment protocol:

- $\quad$ Acetazolamide $250 \mathrm{mg}: 1$ tablet 2 times a day for 3 months with decreasing discontinuation;

- Cotrimoxazole $480 \mathrm{mg}$ injectable: 4 ampoules in $375 \mathrm{ml}$ of $5 \%$ glucose serum, 3 times a day for one (1) month.

- Dexamethasone 4 mg: 4 ampoules per day for one (1) month; Paracetamol perfusable intravenously slowly every hour for 72 hours.

Then a relay was conducted, made on the basis of: Cortancyl 20 $\mathrm{mg}$ at the rate of one (1) tablet once a day in the morning with decreasing discontinuation, Bactrim $960 \mathrm{mg}$ due to two (2) tablets two (2) times a day with the continuation of Acetazolamide at the same dosage for another two (2) months. Optical correction after refractometry was introduced to improve the patient's quality of life, with the following optical parameters:

- OD: Sphere -0.62; Cylinder (-0.25); Axis 108 ${ }^{\circ}$.

- OG: Sphere -0.50; Cylinder (-0.25); Axis $108^{\circ}$.

At three weeks of treatment, the evolution is marked by a clear improvement in the clinical condition of the patient with the disappearance of headaches, vision disorders and hemiparesis. A control brain computed tomography was performed after three (3) weeks showing a decrease in pathological mass.

\section{Discussion}

The Papillary edema is the manifestation of axonal suffering of the ganglion cells of the optic nerve [6]. In this case, it is due to the brain abscess frequently responsible for HIC ??, because of the extensive peri-lesional edema [8]. It is often associated with headache, nausea, vomiting and focal neurological deficits [5]. In this patient, the papillary edema was the evocative of the underlying pathology and pushed us to do more investigations.

According to the literature, cerebral toxoplasmosis is most often related to the endogenous reactivation of parasitic cysts present in the central nervous system (CNS), due to immune deficiency (most often by the human immunodeficiency virus - HIV) [9]. In our case, HIV serology was negative. However, our patient would have undergone long-term antibiotic therapy that could probably be the basis of her immune deficiency. The diagnosis of abcede cerebral toxoplasmosis is based on the realization of brain MRI with injection of contrast medium, failing which a cerebral computed tomography (CT) with contrast medium [5] and the presence of a very high IgG positive Toxoplasma gondii serology in the serum [10]. In this study, MRI and were performed, confirming capsulated abscessed cerebral toxoplasmosis.

Cotrimoxazole is an attractive attack treatment for cerebral toxoplasmosis, available, effective and affordable [11]. As a specific treatment, this patient benefited from Cotrimoxazole injectable and then tablet as a relay. The use of Acetazolamide reduces the production of LCS by choroidal plexuses thus decreases the Intracranial pressure [12]. Our patient benefited from Acetazolamide $250 \mathrm{mg}$ two (2) times a day to reduce headaches, in combination with paracetamol. The prescription of corticosteroid therapy is indicated in patients with symptoms of increased intracranial pressure (headache, vomiting) and in case of chorioretinitis during cerebral toxoplasmosis [4,13]. We used Dexamethasone injection 12 mg daily in this patient followed by Cortancyl $20 \mathrm{mg}$ relay tablet. According to the literature, clinical improvement is rapid, occurring after one to two weeks.

Monitoring by systematic imaging, the improvement of which results in a decrease in the size of the pathological lesion is also observed [14]. For our case the course after 3 weeks was marked by a clear improvement in the clinical condition and a decrease in the pathological mass on computed tomography (Figure 5). Continued treatment was observed for two (2) months.

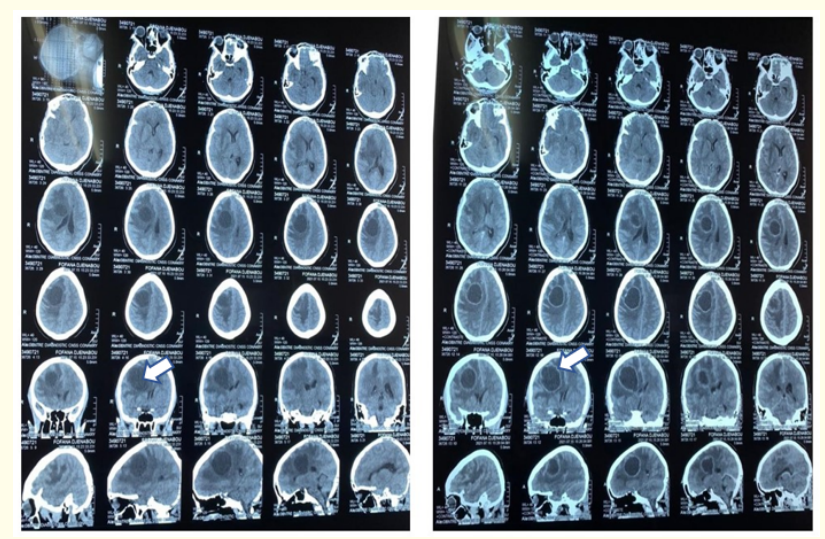

Figure 2: Control computed tomography (CT) scan at day 21 of evolution. The arrow indicates the lesion that has shrunk under treatment. 


\section{Conclusion}

When faced with papillary edema associated with headaches and projectile vomiting in an infectious context, a toxoplasma gondii brain abscess should be suspected until proven otherwise. The positive diagnosis is based on the bundles of clinical and imaging arguments for which the ophthalmologist plays an important role. Its most dreaded complication is irreversible blindness. However, its management is multidisciplinary, so it is important to refer in time to specialized structures for early and rapid management in order to preserve visual function.

\section{Conflicts of Interest}

The authors do not declare any conflict of interest in connection with this work.

\section{Authors' Contribution}

Authors have contributed to one or more levels of manuscript writing from protocol, taking and writing. All of them read and approved the final manuscript.

\section{Bibliography}

1. Killer HE., et al. "Papilledema revisited: is its pathophysiology really understood?" Clinical and Experimental Ophthalmology 37 (2009): 444-447.

2. Bakayoko S., et al. "Bilateral papillary edema secondary to intracranial hypertension in a teenage girl". Pan African Medical Journal 26 (2017): 123.

3. Kamami-Levy C and Vignal-Clermont DC. "Conduct to be held in front of a papillary edema". 8.72 (2014): 57-62.

4. Pearson RD. "Toxoplasmosis - Infectious diseases". Edition Prof Man MSD (2021).

5. Greenlee JE. “Brain abscess - Neurological disorders". Prof Man MSD Edition (2021).

6. Garrity J. "Papillary edema - Eye disorders". Prof Man MSD Edition (2021).

7. Killer HE., et al. "Cerebrospinal fluid dynamics between the intracranial and the sub- arachnoid space of the optic nerve. Is it always bidirectional?" Brain 130.2 (2007): 514-520.

8. F Proust., et al. "Castel. Intracranial hypertension". Neurosurgery Campus (2021).
9. Schmidt M., et al. "Clinical features and outcomes in patients with disseminated toxoplasmosis admitted to intensive care: a multicenter study". Clinical Infectious Diseases 57 (2013): 1535-1541.

10. Tan IL., et al. "HIV-associated opportunistic infections of the CNS”. Lancet Neurology 11 (2012): 605-617.

11. Cabié A. "Cotrimoxazole for therapy of toxoplasmic encephalitis in patients with AIDS". La Lettre de l'Infectiologue 21.5 (2006): 218-221.

12. Timmermans G., et al. "Primary "pseudotumor cerebri" syndrome or idiopathic intracranial hypertension: clinical features and treatment". Revue Médicale de Liège 70.10 (2015): 488-494.

13. Haute Autorité de la Santé (HAS). Diagnostic toxopl asmose biologique de la chez le s immunodéprimé-Argumentaire. Mai (2017).

14. Magalhaes E., et al. "Cerebral toxoplasmosis". Reanimation 24 (2015): 337-340.

\section{Assets from publication with us}

- Prompt Acknowledgement after receiving the article

- Thorough Double blinded peer review

- Rapid Publication

- Issue of Publication Certificate

- High visibility of your Published work

Website: www.actascientific.com/

Submit Article: www.actascientific.com/submission.php

Email us: editor@actascientific.com

Contact us: +919182824667 